\title{
FIR signature verification system characterizing dynamics of handwriting features
}

\author{
Pitak Thumwarin ${ }^{1 *}$, Jitawat Pernwong ${ }^{1}$ and Takenobu Matsuura ${ }^{2}$
}

\begin{abstract}
This paper proposes an online signature verification method based on the finite impulse response (FIR) system characterizing time-frequency characteristics of dynamic handwriting features. First, the barycenter determined from both the center point of signature and two adjacent pen-point positions in the signing process, instead of one pen-point position, is used to reduce the fluctuation of handwriting motion. In this paper, among the available dynamic handwriting features, motion pressure and area pressure are employed to investigate handwriting behavior Thus, the stable dynamic handwriting features can be described by the relation of the time-frequency characteristics of the dynamic handwriting features. In this study, the aforesaid relation can be represented by the FIR system with the wavelet coefficients of the dynamic handwriting features as both input and output of the system. The impulse response of the FIR system is used as the individual feature for a particular signature. In short, the signature can be verified by evaluating the difference between the impulse responses of the FIR systems for a reference signature and the signature to be verified. The signature verification experiments in this paper were conducted using the SUBCORPUS MCYT-100 signature database consisting of 5,000 signatures from 100 signers. The proposed method yielded equal error rate (EER) of $3.21 \%$ on skilled forgeries.
\end{abstract}

\section{Introduction}

Now the need for biometric authentication systems is on the rise because of the ease of use without a password or keycard required. Signature verification is one of the biometrics based on handwriting behavior. In several parts of the world, a signature is the customary way to verify an individual in daily activities, such as withdrawing cash from a bank account and entering a contract. Although visual examination is a popular method for signature verification, it usually takes a long time to process and there are occasions in which examiners make mistakes. To effectively verify the signature, an automatic system for signature verification is required. Signature verification methods are largely classified into two classes: One is the offline method based only on static visual information, and the other is the online method based on the dynamics of handwriting process with a major advantage over the first method in that it is very difficult to forge or copy the dynamics which are invisible. It is well known

\footnotetext{
*Correspondence: ktpitak@kmitl.ac.th

1 Faculty of Engineering, King Mongkut's Institute of Technology Ladkrabang, Ladkrabang, Bangkok 10520, Thailand

Full list of author information is available at the end of the article
}

that handwriting is a highly individual entry depending on such many factors as country, age, habits, psychological or mental state, physical, and practical conditions [1]. For instance, size of scripts, location of scripts, shape of scripts, and duration time in handwriting process of scripts written by the same writer are never precisely the same due to the fluctuation of handwriting. Although the handwriting has many fluctuations described above, it is necessary to extract the feature of handwriting which is more consistent and not likely to be changed, for online signature verification. Such a feature is considered as a stable feature of handwriting. Hence, the preprocessing and feature extraction are very important for online signature verification. Furthermore, in the signature verification, the registration requirement of a large number of signatures of one same individual is impractical. Therefore, to extract the stable handwriting feature from the limited number of signatures is one of the important problems of online signature verification. The issues, i.e., unstable handwriting features and large number of signatures required in the registration process, make the development of online signature verification complicated. Moreover, it is desirable that the feature of handwriting should carry only

\section{是 Springer}

(c) 2013 Thumwarin et al: licensee Springer. This is an Open Access article distributed under the terms of the Creative Commons Attribution License (http://creativecommons.org/licenses/by/2.0), which permits unrestricted use, distribution, and reproduction in any medium, provided the original work is properly cited. 
the essential information of a particular signature and the size of the handwriting feature should be as minimal as possible.

Many methods for online signature verification have been proposed [1-21]. The state-of-the- art online signature verification and literature reviews can be found in [1-5]. In those studies, the individual features of a signature are extracted using the pen-point position, pen pressure, and pen inclination. Many techniques have been used to extract the feature of handwriting and verify the signature, such as DP (Dynamic Programming) matching [6-11], hidden Markov model [12,13], Fourier series [14-16], and support vector machine $[17,18]$. However, in several studies, a linear combination of several features of handwriting was used to verify signature: the suitable weight given to each feature in the linear combination is difficult to determine. Most papers have considered a fusion of independent features, but few papers [14-16] have considered the dynamic relation between the features. In this paper, the barycenter instead of the pen-point position is used to extract the stable handwriting feature. Besides the common handwriting features, i.e., horizontal components, vertical components, and handwriting velocity, two additional dynamic handwriting features were introduced, namely, motion pressure and area pressure, both of which incorporate pen-point pressure and handwriting motion into the handwriting features. Thus, the individual feature of handwriting in the signing process can be extracted by taking into account the dynamic relation between the handwriting features. Moreover, in order to reduce fluctuation of handwriting, stable time-frequency characteristics of the pen-point movement in the signing process are determined from the wavelet expansion of the handwriting features. In this study, the aforementioned dynamic relation can be represented by the finite impulse response (FIR) system with the determined wavelet coefficients of the handwriting features as both input and output. That is to say, the impulse response of the FIR system can be characterized not only by the handwriting features but also the dynamic relation between them. In our method, the obtained impulse response is used as the individual handwriting feature for signature verification. In this paper, the signature verification problem can be reduced to system identification problem. The remaining of the paper is organized as follows. Section 2 presents preprocessing process. In this section, a normalization of the size and location of signature are performed. The barycenter trajectory is introduced to use instead of pen-point position to reduce a sensitivity of handwriting motion. In Section 3, new signing features barycenter trajectory, area pressure and motion pressure are introduced. Then the time-frequency characteristics of the signing feature are determined to extract the stable handwriting feature in

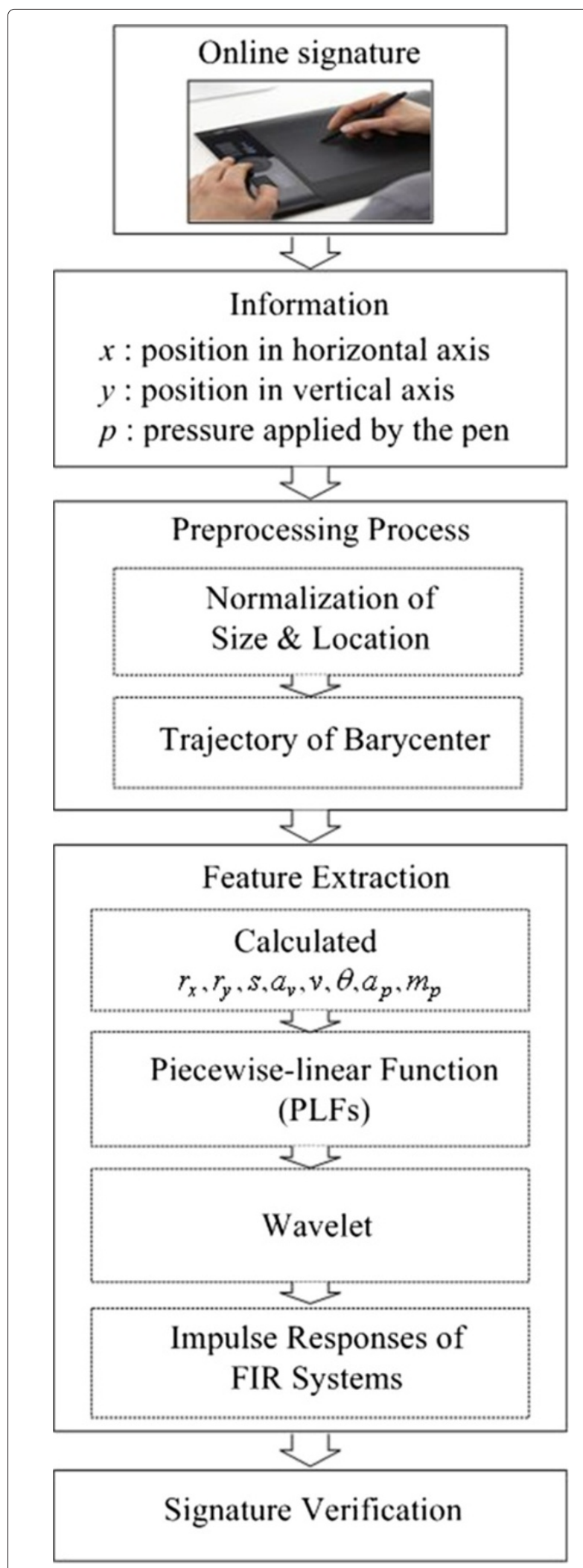

Figure 1 Scheme of an automatic system for signature verification. 
the signing process. Moreover, the FIR system characterizing the handwriting system is introduced. The obtained impulse response of the FIR system is used as the unique feature for signature verification. In Section 4, the signature verification algorithm is presented. In Section 5, the experimental results are given to show the effectiveness of the proposed method. Section 6 is the conclusion. Figure 1 shows scheme of the proposed signature verification system.

\section{Preprocessing}

In this paper, signatures are written on a graphical tablet. The horizontal component, vertical component, and pen pressure of pen-point position at a time, $t=n \tau\left(\equiv t_{n}\right)$, in the signing process are represented as $x\left(t_{n}\right), y\left(t_{n}\right)$, and $p\left(t_{n}\right)$ respectively, where $\tau$ is a constant sampling rate. In order to reduce the fluctuation of handwriting, three types of normalization with respect to size, location, and duration time in the signing process are performed with the details described in the following subsections.

\subsection{Normalization of size}

The size of a signature is standardized after removing the duplicated points of pen-point position. In order to standardize the size of signature, the horizontal component,

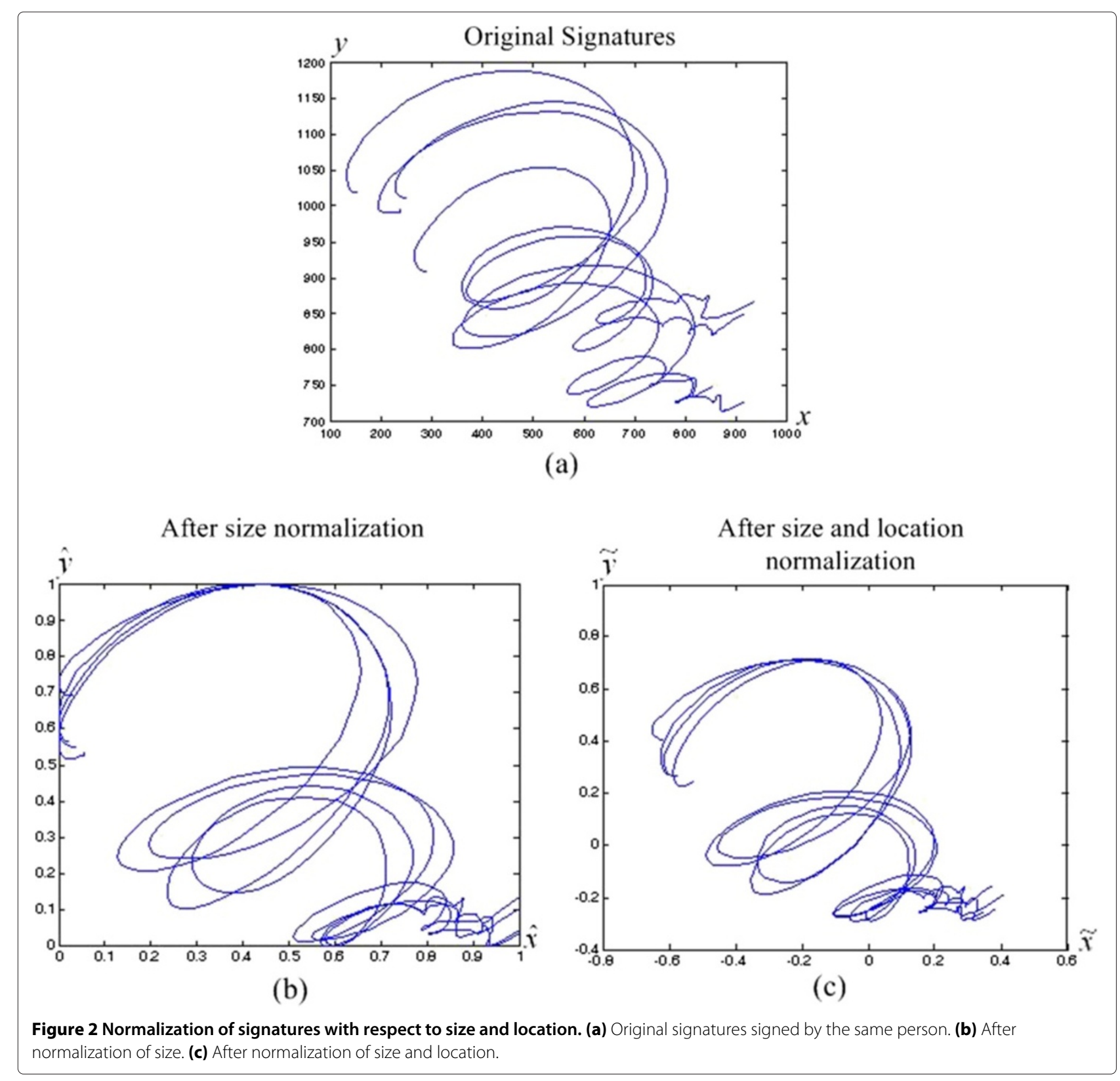


vertical component, and pen pressure $x\left(t_{n}\right), y\left(t_{n}\right)$, and $p\left(t_{n}\right)$ are normalized as

$$
\hat{z}\left(t_{n}\right)=\frac{z\left(t_{n}\right)-z_{\min }}{z_{\max }-z_{\min }},(z=x, y, p)
$$

where

$$
\begin{aligned}
z_{\min } & =\min _{n \in\{0, \cdots, N-1\}} z\left(t_{n}\right), \\
z_{\max } & =\max _{n \in\{0, \cdots, N-1\}} z\left(t_{n}\right),
\end{aligned}
$$

and $N$ is the total number of sampled points of pen-point positions.

\subsection{Normalization of location}

The location of the signature is normalized as

$$
\tilde{z}\left(t_{n}\right)=\hat{z}\left(t_{n}\right)-c_{z},(z=x, y)
$$

where $c_{z}$ is the center point of script computed from

$$
c_{z}=\frac{1}{N} \sum_{n=0}^{N-1} \hat{z}\left(t_{n}\right)
$$

The example of normalization with respect to size and location is shown in Figure 2. It can be seen from Figure 2 that the fluctuation of size and location of the signatures can be reduced.

\subsection{Trajectory of barycenter}

In order to obtain the stable handwriting motion in signing process, the trajectory of the barycenter determined from the center point of a script and the two adjacent penpoint positions with respect to time in signing process is used in place of pen-point trajectory. The barycenter coordinates $\left(r_{x}\left(t_{n}\right), r_{y}\left(t_{n}\right)\right)$ shown in Figure 3 are calculated by the following equations:

$$
r_{x}\left(t_{n}\right)=\frac{\tilde{x}\left(t_{n}\right)+\tilde{x}\left(t_{n+1}\right)}{3}, r_{y}\left(t_{n}\right)=\frac{\tilde{y}\left(t_{n}\right)+\tilde{y}\left(t_{n+1}\right)}{3},
$$

where $n=0,1,2, \ldots, N-1$. The effectiveness of using the barycenter to reduce the fluctuation of handwriting motion has been reported in our earlier work [19].

\section{Feature extraction}

\subsection{Dynamic handwriting features}

It is assumed here that the individual signing feature can be described by the following eight features:

- Horizontal direction $r_{x}\left(t_{n}\right)$ of the barycenter trajectory in signing process.

- Vertical direction $r_{y}\left(t_{n}\right)$ of the barycenter trajectory in signing process.

- Areal velocity $a_{\nu}\left(t_{n}\right)$, which is the area swept out per unit time by the pen-point moving along the barycenter trajectory of handwriting script. The $a_{v}\left(t_{n}\right)$ is computed from

$$
\begin{array}{r}
a_{v}\left(t_{n}\right)=\frac{1}{2}\left|\begin{array}{cc}
r_{x}\left(t_{n-1}\right) & r_{y}\left(t_{n-1}\right) \\
r_{x}\left(t_{n}\right) & r_{y}\left(t_{n}\right)
\end{array}\right|, \\
(n=1,2, \ldots, N-1)
\end{array}
$$

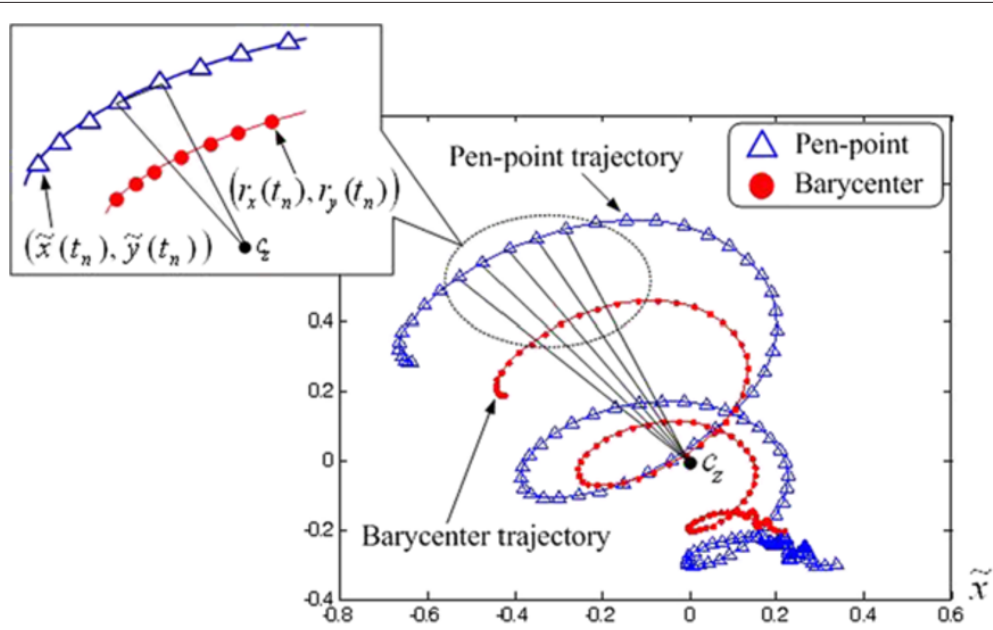

Figure 3 Trajectories of pen-point position and barycenter of signature. 

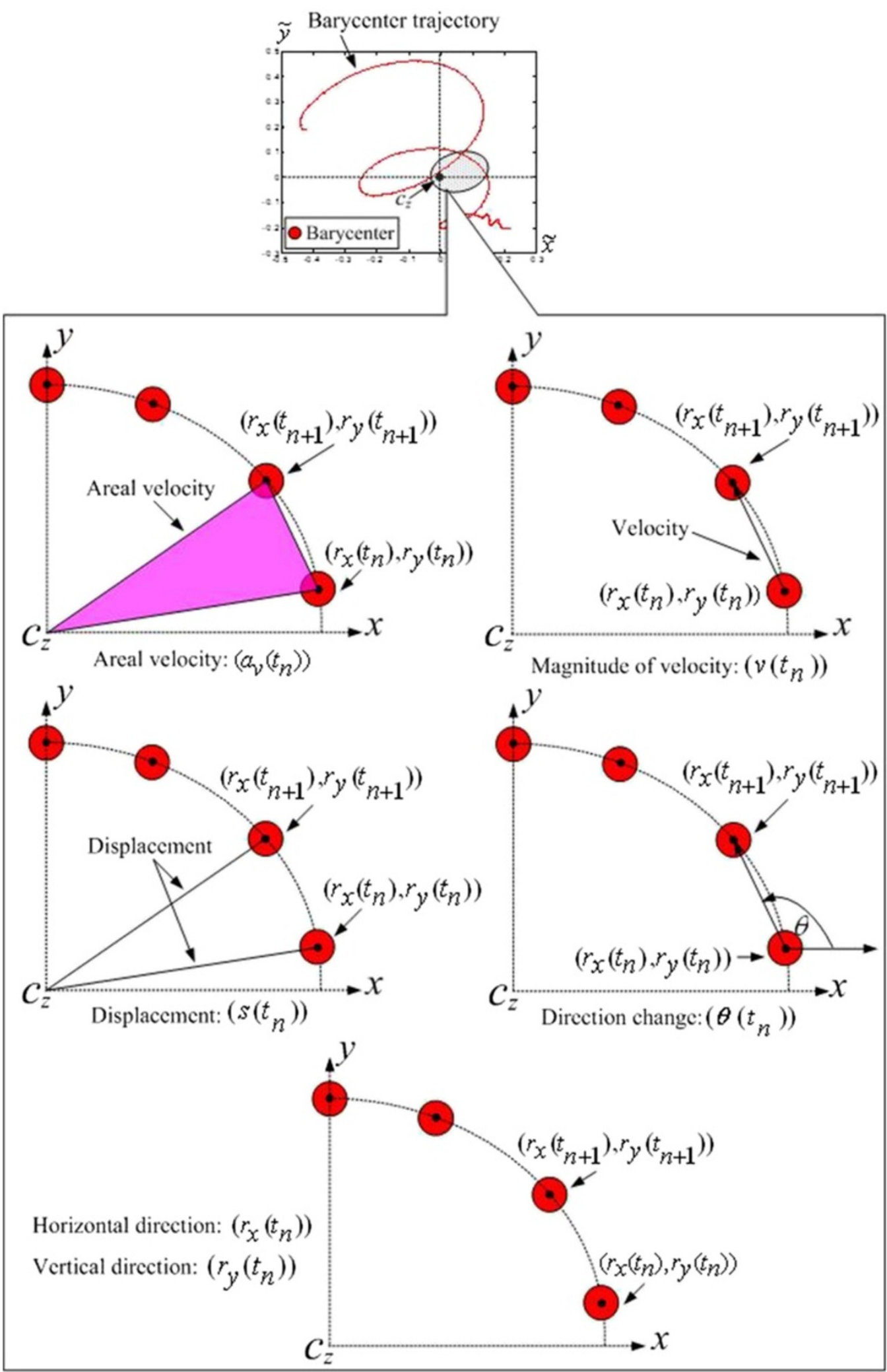

Figure 4 The six features related to the horizontal and vertical components. 
- Displacement $s\left(t_{n}\right)$, which is the distance from the center of signature to barycenter trajectory at time $t_{n}$. The $s\left(t_{n}\right)$ can be calculated as

$$
\begin{array}{r}
s\left(t_{n}\right)=\sqrt{r_{x}\left(t_{n}\right)^{2}+r_{y}\left(t_{n}\right)^{2}}, \\
(n=0,1,2, \ldots, N-1)
\end{array}
$$

- Magnitude of velocity $v\left(t_{n}\right)$ of the barycenter trajectory. The $v\left(t_{n}\right)$ is computed from

$$
\begin{array}{r}
v\left(t_{n}\right)=\sqrt{\left(\Delta x\left(t_{n}\right)\right)^{2}+\left(\Delta y\left(t_{n}\right)\right)^{2},} \\
(n=0,1,2, \ldots, N-1)
\end{array}
$$

where

$$
\begin{aligned}
& \Delta x\left(t_{n}\right)=r_{x}\left(t_{n+1}\right)-r_{x}\left(t_{n}\right), \\
& \Delta y\left(t_{n}\right)=r_{y}\left(t_{n+1}\right)-r_{y}\left(t_{n}\right)
\end{aligned}
$$

- The direction change $\theta\left(t_{n}\right)$ of the barycenter trajectory. The $\theta\left(t_{n}\right)$ can be calculated by

$$
\begin{array}{r}
\theta\left(t_{n}\right)=\tan ^{-1}\left(\frac{r_{y}\left(t_{n+1}\right)-r_{y}\left(t_{n}\right)}{r_{x}\left(t_{n+1}\right)-r_{x}\left(t_{n}\right)}\right), \\
(n=0,1,2, \ldots, N-1)
\end{array}
$$

Examples of the above six features are shown in Figure 4.

It is known that the pen pressure is one of the important features for signature verification. In this paper, two features related to the pen pressure are introduced as follows:

- Area pressure $a_{p}\left(t_{n}\right)$ as shown in Figure $5 \mathrm{~b}$, which is the area of triangle consisting of pen pressure, pen-point position, and center of signature in the signing process. The $a_{p}\left(t_{n}\right)$ is computed from

$$
\begin{aligned}
& a_{p}\left(t_{n}\right)=\frac{1}{2} \times \hat{p}\left(t_{n}\right) \times \sqrt{\tilde{x}\left(t_{n}\right)^{2}+\tilde{y}\left(t_{n}\right)^{2}}, \\
&(n=0,1,2, \ldots, N-1)
\end{aligned}
$$

- Motion pressure $m_{p}\left(t_{n}\right)$ as shown in Figure $5 \mathrm{~b}$, which is distance of the diagonal between two adjacent pen-point positions and the pen pressure. The $m_{p}\left(t_{n}\right)$ is computed from

$$
m_{p}\left(t_{n}\right)=\sqrt{\hat{p}\left(t_{n}\right)^{2}+S\left(t_{n}\right)^{2}}
$$

where

$$
\begin{aligned}
\Delta x\left(t_{n}\right) & =\hat{x}\left(t_{n+1}\right)-\hat{x}\left(t_{n}\right), \\
\Delta y\left(t_{n}\right) & =\hat{y}\left(t_{n+1}\right)-\hat{y}\left(t_{n}\right), \\
\Delta p\left(t_{n}\right) & =\tilde{p}\left(t_{n+1}\right)-\tilde{p}\left(t_{n}\right), \\
S\left(t_{n}\right) & =\sqrt{\left(\Delta x\left(t_{n}\right)\right)^{2}+\left(\Delta y\left(t_{n}\right)^{2}\right)+\left(\Delta p\left(t_{n}\right)^{2}\right)}
\end{aligned}
$$

Figure 5 shows the area pressure $a_{p}\left(t_{n}\right)$ and the motion pressure $m_{p}\left(t_{n}\right)$ in the signing process determined by using Equations 9 and 10.

In this paper, in order to normalize the duration time in signing process, the piecewise-linear functions (PLFs) of $r_{x}\left(t_{n}\right), r_{y}\left(t_{n}\right), s\left(t_{n}\right), a_{v}\left(t_{n}\right), v\left(t_{n}\right), \theta\left(t_{n}\right), a_{p}\left(t_{n}\right)$, and $m_{p}\left(t_{n}\right)$ are determined by connecting two adjacent components with a straight line. The PLFs can be described as

$$
\begin{aligned}
q(t) & =\sum_{n=0}^{N-1} q\left(t_{n}\right) \phi_{n}(t), \quad(t \in T), \\
(q & \left.=r_{x}, r_{y}, s, a_{v}, v, \theta, a_{p}, m_{p}\right)
\end{aligned}
$$

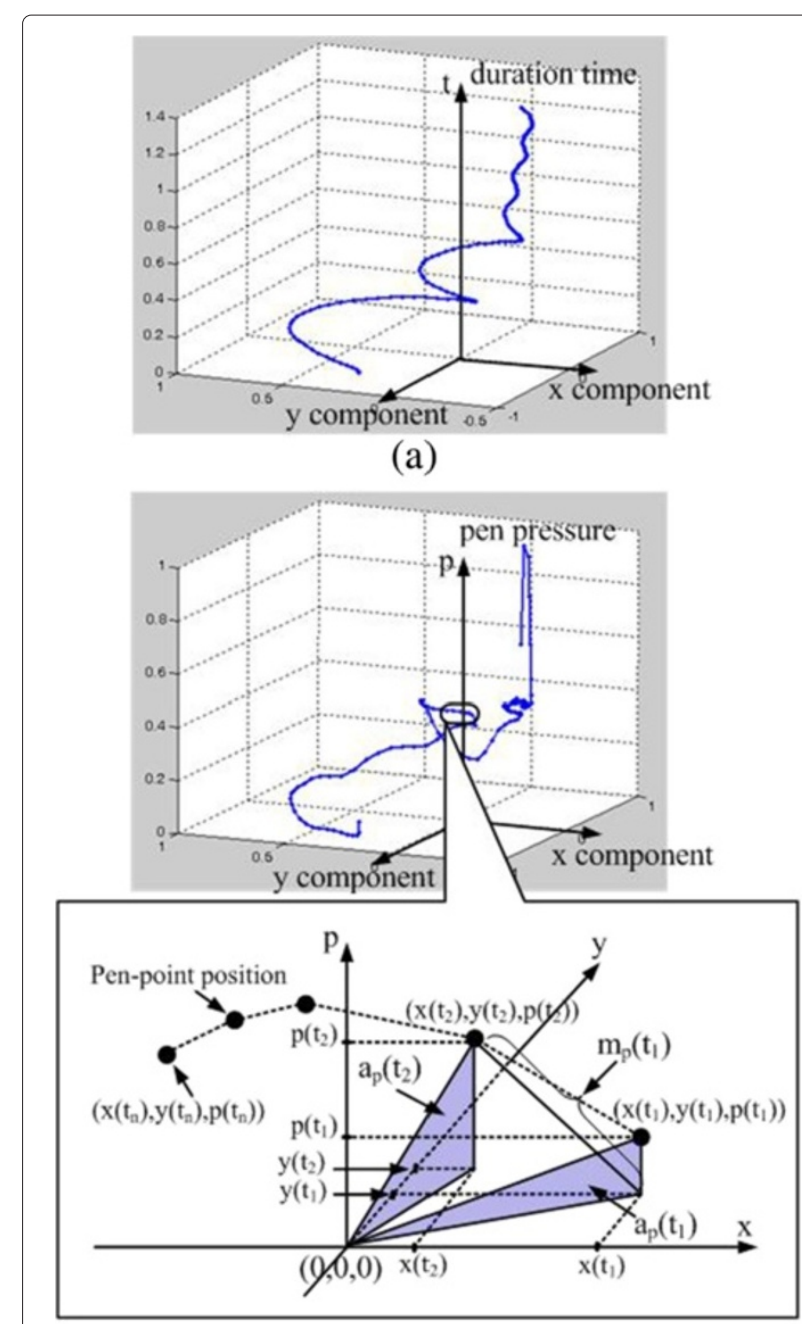

(b)

Figure 5 Area pressure $a_{p}\left(t_{n}\right)$ and the motion pressure $m_{p}\left(t_{n}\right)$ in the signing process. (a) The plot of $x, y$ components and duration time of the signing process. (b) The plot of area pressure $a_{p}\left(t_{n}\right)$ and motion pressure $m_{p}\left(t_{n}\right)$. 
where

$$
\phi_{n}(t)=\left\{\begin{array}{cc}
\frac{t-t_{(n-1)}}{t_{n}-t_{(n-1)}}, & t \in\left[t_{(n-1)}, t_{n}\right] \\
\frac{t_{(n+1)}-t}{t_{(n+1)}-t_{n}}, & t \in\left[t_{n}, t_{(n+1)}\right], \\
0, & t \notin\left[t_{(n-1)}, t_{(n+1)}\right]
\end{array}\right.
$$

and $T$ is the duration time of signing process. Using Equation 12, the duration time is normalized as $T=$ $T_{\text {norm}}$. The $T_{\text {norm }}$ is determined by the training data for a particular signature. Examples of duration time normalization are shown in Figure 6. It can be seen from Figure 6 that the fluctuation in the duration time in the signing process can be reduced after normalization.

Figure 7 shows the dynamic handwriting features obtained from genuine signatures and forgeries for person A. It can be seen from Figure 7 that the dynamic handwriting features obtained from genuine signatures are different from those of forgeries.

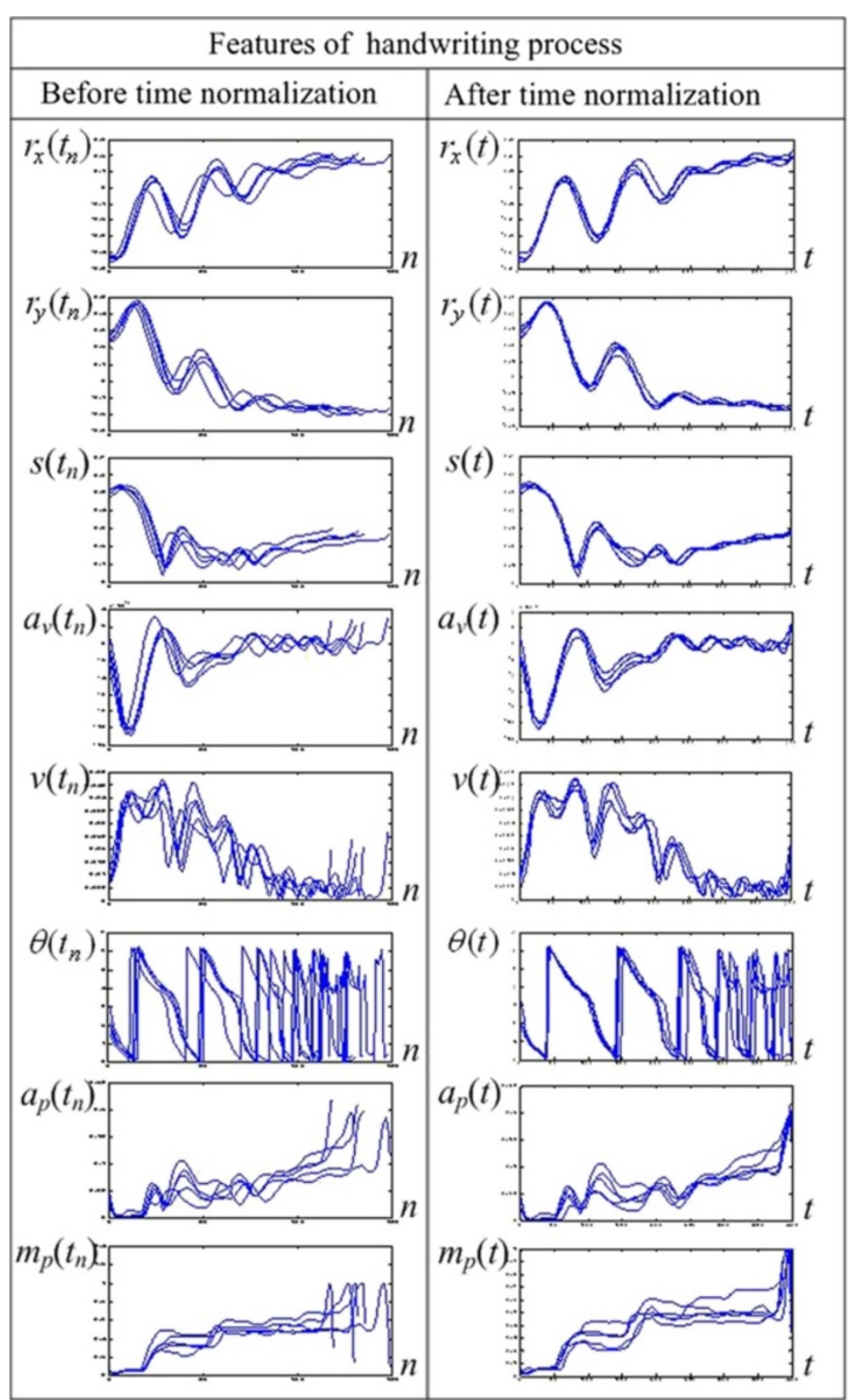

Figure 6 Examples of time normalization. 


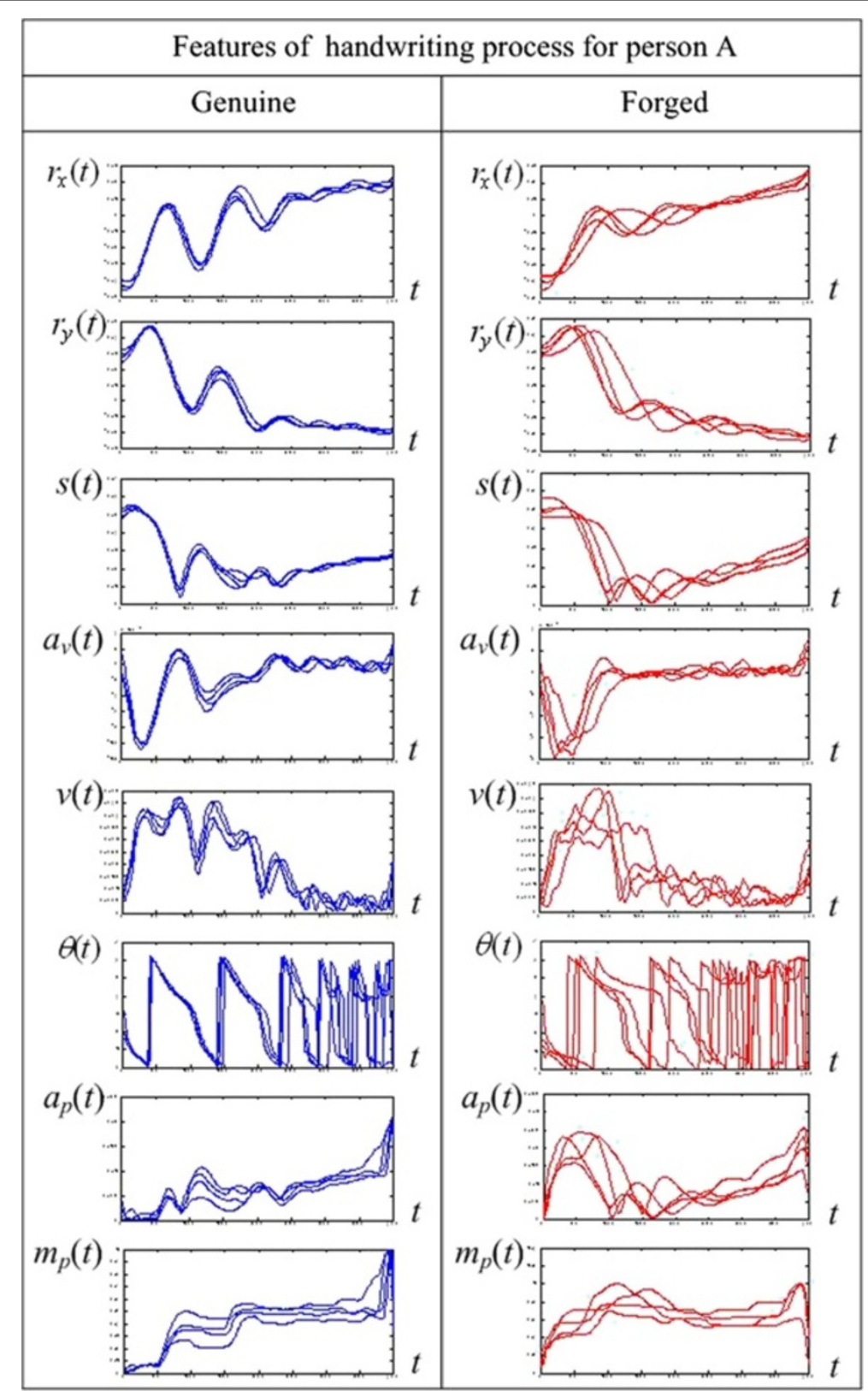

Figure 7 Examples of the signing features obtained from genuine signatures and forgeries.

\subsection{Time-frequency characteristic of the dynamic handwriting features}

Wavelet transforms were used for signature verifications in the works [22-24]. However, they are not taken into account the time-frequency characteristics of the handwriting feature extracting the stable handwriting features. The time-frequency characteristics of the dynamic handwriting features in the signing process depend on the signing style of a particular writer. In other words, the time-frequency characteristics of the dynamic handwriting features in signing process represent an individual feature for signature verification. In order to extract the individual signing feature, the eight features $r_{x}(t), r_{y}(t), s(t), a_{v}(t), v(t), \theta(t), a_{p}(t)$, and $m_{p}(t)$ obtained in the preceding section are expressed by an orthonormal wavelet $\psi_{k, l}(t)$ as

$$
\begin{aligned}
q(t) & =\sum_{k=0}^{2^{z}-1} a_{z}^{(q)}(k) \varphi_{k, z}(t)+\sum_{l=z}^{L-1} \sum_{k=0}^{2^{l}-1} d_{l}^{(q)}(k) \psi_{k, l}(t), \\
(q & \left.=r_{x}, r_{y}, s, a_{v}, v, \theta, a_{p}, m_{p} ; z=0,1,2, \ldots, L-1\right),
\end{aligned}
$$


where

$$
a_{z}^{(q)}(k)=\left\langle q, \varphi_{k, z}(t)\right\rangle, d_{l}^{(q)}(k)=\left\langle q, \psi_{k, l}(t)\right\rangle
$$

$L$ is maximum number of level of the time-frequency characteristics. The $\psi_{k, l}(t)$ and $\varphi_{k, z}(t)$ are the orthonormal Haar wavelet, and the scaling function as defined by the following equations:

$$
\begin{aligned}
\psi_{k, l}(t) & =2^{\frac{l}{2}} \psi\left(2^{l} t-k\right), \\
\left\langle\psi_{l, k}, \psi_{m, n}\right\rangle & =\int_{-\infty}^{\infty} \psi_{l, k}(t) \psi_{m, n}(t) d t=\delta_{l, k} \delta_{m, n}, \\
\varphi_{k, z}(t) & =2^{\frac{z}{2}} \varphi\left(2^{z} t-k\right),(z, k \in Z)
\end{aligned}
$$

where $\delta_{l, k}$ is Kronecker's delta and $Z$ is a set of integers. The $a_{z}^{(q)}(k)$ and $d_{l}^{(q)}(k)$ represent the feature related to the time-frequency characteristics of the dynamic handwriting features. Hence, in order to describe the timefrequency characteristics of the dynamic handwriting features in the signing process, the following vectors are defined as

$$
\begin{aligned}
\mathbf{c}_{z}^{\left(q_{1}\right)} & =\left[c_{z}^{\left(q_{1}\right)}(0), c_{z}^{\left(q_{1}\right)}(1), \ldots, c_{z}^{\left(q_{1}\right)}(\underline{N})\right]^{\mathrm{T}}, \\
\left(q_{1}\right. & \left.=r_{x}, a_{v}, \theta, a_{p} ; \mathbf{c}=a, d\right), \\
\mathbf{c}_{z}^{\left(q_{2}\right)} & =\left[c_{z}^{\left(q_{2}\right)}(0), c_{z}^{\left(q_{2}\right)}(1), \ldots, c_{z}^{\left(q_{2}\right)}(\underline{N})\right]^{\mathrm{T}}, \\
\left(q_{2}\right. & \left.=r_{y}, s, v, m_{p} ; \mathbf{c}=a, d\right), \\
\underline{N} & =2^{(z-1)},
\end{aligned}
$$

where $\mathbf{c}_{z}^{\left(q_{1}\right)}$ and $\mathbf{c}_{z}^{\left(q_{2}\right)}$ are parameter vectors representing the time-frequency characteristics of the dynamic handwriting features. Figure 8 shows example of the wavelet coefficients of time-frequency characteristics $\left(a_{z}^{(q)}, d_{l}^{(q)}\right)$ obtained from two persons. It can be seen from Figure 8 that both $a_{z}^{(q)}$ and $d_{l}^{(q)}$ can extract the stable handwriting feature $\left(a_{4}^{\left(r_{x}\right)}, d_{3}^{(s)}\right)$. In this paper, the stable handwriting features obtained from $a_{z}^{(q)}$ and $d_{l}^{(q)}$ are used for signature verification.

\subsection{FIR system characterizing the dynamics of handwriting features}

In this subsection, a handwriting system is defined as a system characterizing a dynamic feature of handwriting in the signing process. Such a handwriting system is realized by the FIR system which characterizes the timefrequency characteristics of dynamic handwriting features in the signing process. In this study, the wavelet coefficients of the dynamic handwriting features obtained in the preceding section are used as the input and output of the FIR system. The effectiveness of using the FIR system characterizing the handwriting feature for character recognition was reported in our work [25]. Figure 9 shows the FIR system in representing the time-frequency characteristics of handwriting features. $f(m), g(m)$ are the input and output of the FIR system, respectively; $\hat{g}(m)$ is an approximation of $g(m)$; $e(m)$ is error of the approximation.

The FIR system is designed as follows:

First, the FIR system can be described by

$$
\begin{aligned}
\widehat{c}_{z}^{\left(r_{y}\right)}(k) & =\sum_{m=0}^{M} h_{r_{x}, r_{y}}^{\left(c_{z}\right)}(m) c_{z}^{\left(r_{x}\right)}(k-m), \\
\widehat{c}_{z}^{(s)}(k) & =\sum_{m=0}^{M} h_{a_{v}, s}^{\left(c_{z}\right)}(m) c_{z}^{\left(a_{v}\right)}(k-m), \\
\widehat{c}_{z}^{(v)}(k) & =\sum_{m=0}^{M} h_{\theta, v}^{\left(c_{z}\right)}(m) c_{z}^{(\theta)}(k-m), \\
\widehat{c}_{z}^{\left(m_{p}\right)}(k) & =\sum_{m=0}^{M} h_{a_{p}, m_{p}}^{\left(c_{z}\right)}(m) c_{z}^{\left(a_{p}\right)}(k-m), \\
(k & =0,1,2, \ldots, K-1),
\end{aligned}
$$

where $c_{z}^{\left(q_{1}\right)},\left(q_{1}=r_{x}, a_{v}, \theta, a_{p}\right)$ and $c_{z}^{\left(q_{2}\right)},\left(q_{2}=r_{y}, s, v, m_{p}\right)$ are the input and output of the FIR system, respectively, both of which are the wavelet coefficients of the corresponding dynamic handwriting features. $\hat{c}_{z}^{\left(q_{2}\right)}(k)$ is an approximation of $c_{z}^{\left(q_{2}\right)}(k) . M$ is the order of the FIR system. $K$ is the total number of the input and output sequences of the FIR system. $\mathbf{h}_{i, j}^{\left(c_{z}\right)^{\mathrm{T}}}=[h(0)$, $h(1), \ldots, h(M)],\left(i=r_{x}, a_{v}, \theta, a_{p} ; j=r_{y}, s, v, m_{p}\right)$ is the impulse responses of the FIR system. $\mathbf{h}_{i, j}^{\left(c_{z}\right)^{\mathrm{T}}}$ is the transposition of $\mathbf{h}_{i, j}^{\left(c_{z}\right)}$. The impulse response $\mathbf{h}_{i, j}^{\left(c_{z}\right)}$ can be determined in the least-square sense.

$$
\begin{aligned}
E^{\left(c_{z}^{r_{y}}\right)} & =\sum_{k=0}^{K-1} \mid c_{z}^{\left(r_{y}\right)}(k)-\hat{c}_{z}^{\left(r_{y}\right)}\left(k ;\left.\mathbf{h}_{r_{x}, r_{y}}^{\left(c_{z}\right)}\right|^{2} \rightarrow \min ,\right. \\
E^{\left(c_{z}^{s}\right)} & =\sum_{k=0}^{K-1}\left|c_{z}^{(s)}(k)-\hat{c}_{z}^{(s)}\left(k ; \mathbf{h}_{a_{v}, s}^{\left(c_{z}\right)}\right)\right|^{2} \rightarrow \min , \\
E^{\left(c_{z}^{\nu}\right)} & =\sum_{k=0}^{K-1}\left|c_{z}^{(v)}(k)-\hat{c}_{z}^{(v)}\left(k ; \mathbf{h}_{\theta, v}^{\left(c_{z}\right)}\right)\right|^{2} \rightarrow \min , \\
E^{\left(c_{z} m_{p}\right)} & =\sum_{k=0}^{K-1}\left|c_{z}^{\left(m_{p}\right)}(k)-\hat{c}_{z}^{\left(m_{p}\right)}\left(k ; \mathbf{h}_{a_{p}, m_{p}}^{\left(c_{z}\right)}\right)\right|^{2} \rightarrow \min ,
\end{aligned}
$$




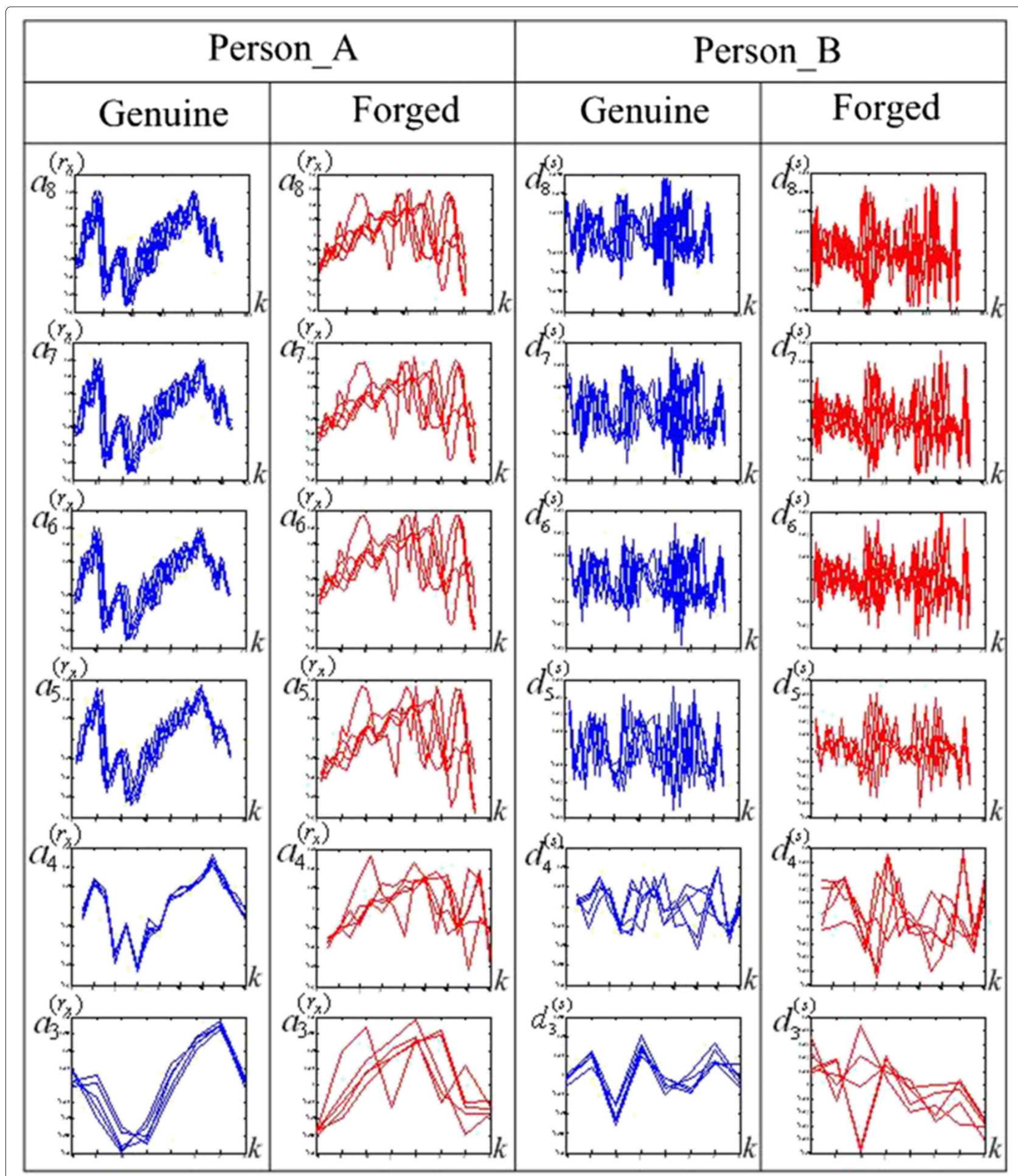

Figure 8 Wavelet coefficients in different levels obtained from genuine signatures and forgeries.

where $\hat{c}_{z}^{\left(r_{y}\right)}\left(k ; \mathbf{h}_{r_{x}, r_{y}}^{\left(c_{z}\right)}\right), \hat{c}_{z}^{(s)}\left(k ; \mathbf{h}_{a_{v}, s}^{\left(c_{z}\right)}\right), \hat{c}_{z}^{(v)}\left(k ; \mathbf{h}_{\theta, v}^{\left(c_{z}\right)}\right)$, and $\hat{c}_{z}^{\left(m_{p}\right)}$ $\left(k ; \mathbf{h}_{a_{p}, m_{p}}^{\left(c_{z}\right)}\right)$ are the approximation of $c_{z}^{\left(r_{y}\right)}\left(k ; \mathbf{h}_{r_{x}, r_{y}}^{\left(c_{z}\right)}\right)$, $c_{z}^{(s)}\left(k ; \mathbf{h}_{a_{v}, s}^{\left(c_{z}\right)}\right), c_{z}^{(v)}\left(k ; \mathbf{h}_{\theta, v}^{\left(c_{z}\right)}\right)$, and $c_{z}^{\left(m_{p}\right)}\left(k ; \mathbf{h}_{a_{p}, m_{p}}^{\left(c_{z}\right)}\right)$, respectively.
The details on how to determine the impulse response are reported in Thumwarin's work [15]. Figure 10 shows the impulse responses of the FIR system characterizing individual dynamics in the signing process, which are 


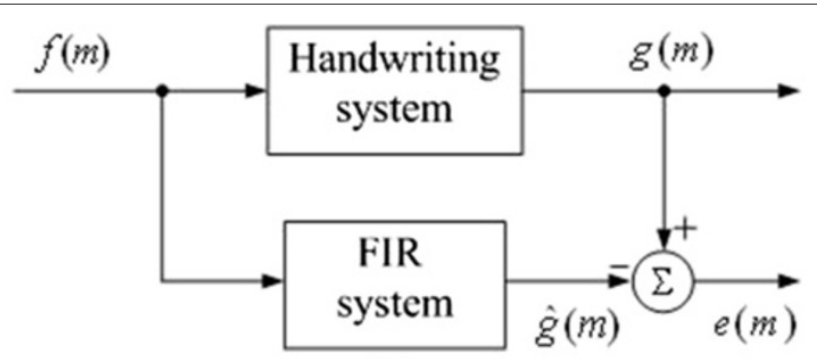

(a)

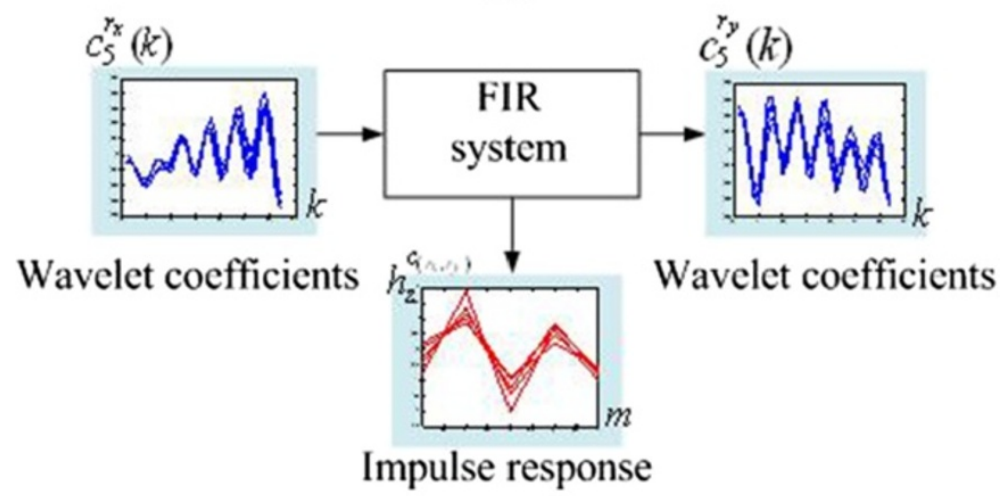

(b)

Figure 9 FIR system in representing the time-frequency characteristics of handwriting features. (a) The FIR system representing the handwriting system. (b) The FIR system characterizing dynamics of handwriting features.

obtained from the genuine signatures and forgeries. It can be seen from Figure 10 that the impulse responses of the FIR system obtained from genuine signatures are quite similar while those obtained from forgeries are different. Therefore, the obtained impulse responses represent the handwriting dynamics and can be used as the individual feature of a particular signature.

\section{Signature verification}

In this section, the impulse responses obtained in the preceding section are used to verify signature. The algorithm is given as follows.

\subsection{Training process}

The following are the steps in the training process:

- First, the member register their name and sign their signatures five times used for reference data and training process.

- Second, the obtained signatures are preprocessed using method in Section 2.

- Third, the eight dynamic handwriting features as described in Section 3.1 are calculated.

- Fourth, after normalization of duration time, they are expanded into wavelet series to determine the time-frequency characteristics of their features. In this step, the suitable level of the time-frequency characteristics which represent the stability of their handwriting feature is determined by using the standard deviation of each level. We select the level of their standard deviations less than the predetermined threshold values as the stable handwriting feature. Therefore, these parameters are the individual features for a particular person.

- Fifth, the obtained time-frequency characteristics of the eight features are used to determine the handwriting system using the FIR systems as described in Section 3.3. In this case, the order of the FIR systems $(M)$ for each particular person can be determined by using standard deviation of the impulse responses. We choose the maximum value of the parameter $M$ that its standard deviation is less than the predetermined threshold value. Then, the obtained impulse responses are combined as

$$
\begin{array}{r}
\mathbf{h}_{i, j}^{(z)^{(\mathrm{ref})}}=\left[\mathbf{h}_{i, j}^{\left(a_{z}\right)^{\mathrm{T}}} \mathbf{h}_{i, j}^{\left(d_{z}\right)^{\mathrm{T}}}\right], \\
\left(i=r_{x}, a_{v}, \theta, a_{p} ; j=r_{y}, s, v, m_{p}\right) .
\end{array}
$$

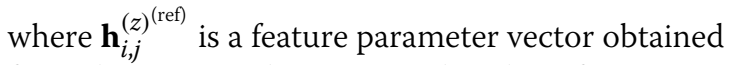
from the registered person used as the reference 


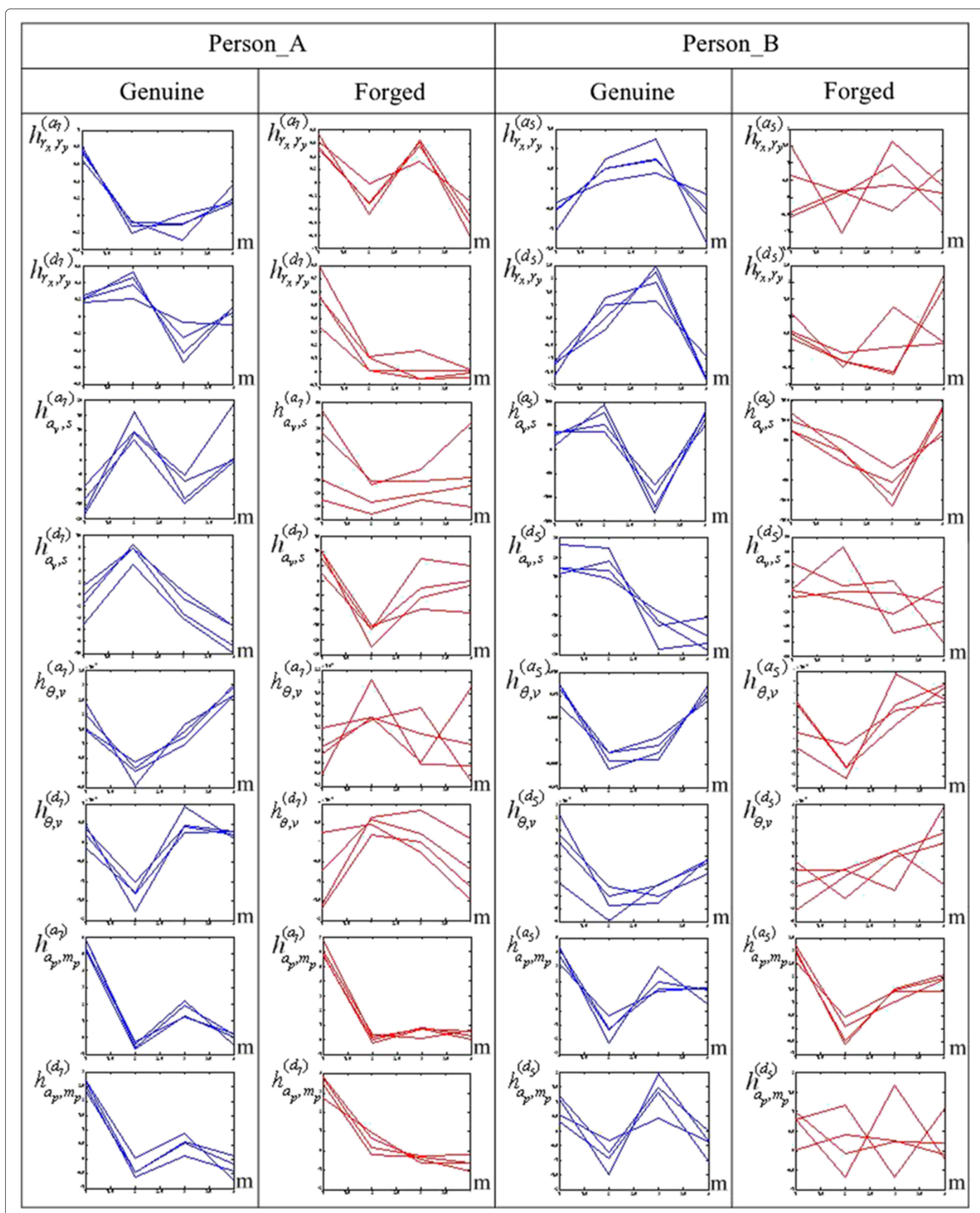

Figure 10 The impulse responses of FIR system obtained from genuine signatures and forgeries. 
handwriting feature. Then the above feature parameter vectors are combined as

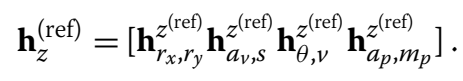

The obtained feature vector $\left(\mathbf{h}_{z}^{(\text {ref })}\right)$, level of the time-frequency characteristics, and order of the FIR system are used as the individual feature for signature verification for a particular person.

\subsection{Testing process}

In the testing process, the following steps must be undertaken:

- First, test persons put their registered name and sign their signatures.

- Second, the test signature is preprocessed using the method in Section 2.

- Third, after normalization of duration time and using the registered name corresponding to the test name, the number level of the registered person is used to determine the time-frequency characteristics of their features.

- Fourth, the obtained time-frequency characteristics of the eight features are used to determine the handwriting system using the FIR systems as described in Section 3.3. In this case, the order of the FIR systems $(M)$ of the registered name corresponding to the test name is used to calculate the impulse responses. Then the obtained impulse responses are combined as

$$
\begin{array}{r}
\mathbf{h}_{i, j}^{(z)^{(\text {test })}}=\left[\mathbf{h}_{i, j}^{\left(a_{z}\right)^{\mathrm{T}}} \mathbf{h}_{i, j}^{\left(d_{z}\right)^{\mathrm{T}}}\right], \\
\left(i=r_{x}, a_{v}, \theta, a_{p} ; j=r_{y}, s, v, m_{p}\right) .
\end{array}
$$

where $\mathbf{h}_{i, j}^{(z)^{\text {(test) }}}$ is a feature parameter vector obtained from the tested person used as the tested handwriting feature. Then the above feature parameter vectors are combined as

$$
\mathbf{h}_{z}^{\text {(test) }}=\left[\mathbf{h}_{r_{x}, r_{y}}^{z^{\text {(test) }}} \mathbf{h}_{a_{v}, s}^{z^{\text {(test) }}} \mathbf{h}_{\theta, v}^{z^{\text {(test) }}} \mathbf{h}_{a_{p}, m_{p}}^{z^{\text {(test) }}}\right]
$$

Then the tested signature is verified as

$$
\begin{aligned}
& \text { true if } \| \mathbf{h}_{\mathbf{z}}{ }^{{ }^{(\text {ref })}-\mathbf{h}_{\mathbf{z}}{ }^{\text {(test) }} \|<\eta} \text {, } \\
& \text { false otherwise, }
\end{aligned}
$$

where $\|\cdot\|$ is Euclidean norm, and $\eta$ is a predetermined threshold value determined by using the experiments with the training data for a particular signature.

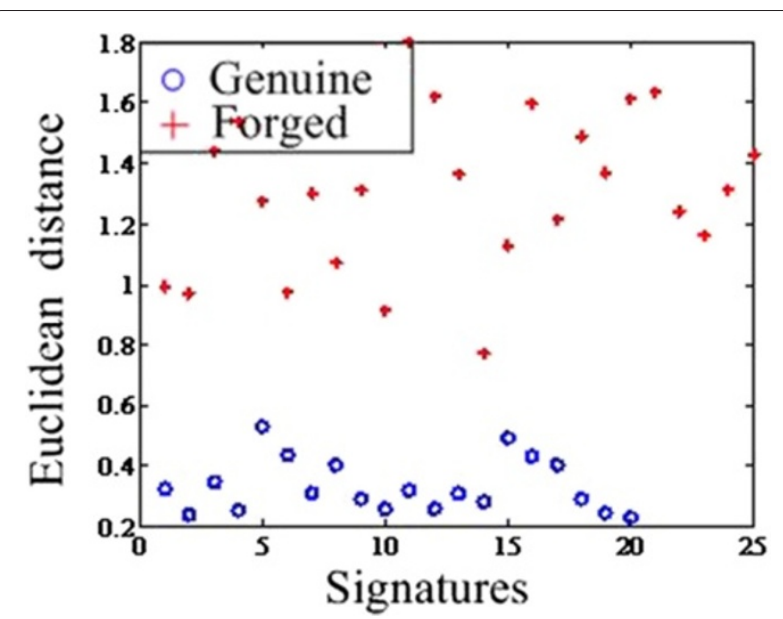

Figure 11 The plot of Euclidean distance obtained from the genuine signatures and forgeries.

Examples of the Euclidean distances between the feature vector $\mathbf{h}_{\mathbf{z}}{ }^{\text {(ref) }}$ and $\mathbf{h}_{\mathbf{z}}{ }^{\text {(test) }}$ obtained from genuine signatures and forgeries are shown in Figure 11. It can be seen from Figure 11 that the Euclidean distances obtained from genuine signatures are less than those obtained from the forgeries. That is to say, Figure 11 shows that individual handwriting features with respect to the change of shape, velocity, and pressure during signing process are clearly unique. This shows that the signature can be identified by the proposed handwriting features.

\section{Experimental result}

The experiments were conducted using the MCYT-100 [26] public signature database. The database consists of 100 signers, each of whom supplies 25 genuine signatures and another 25 forged signatures. In our experiments, five genuine signatures were randomly selected and used as the reference and training data for a particular signature. The comparison of the experimental results obtained from the proposed method and some related works that used MCYT-100 is shown in Table 1. It can be seen from Table 1 that the result obtained from the proposed method is better than those of the other methods.

Table 1 Comparison of proposed method with other works Reference Equal error rate (EER in \%)

\begin{tabular}{lc} 
& Skilled forgery \\
\hline Proposed method & 3.21 \\
Work [20] & 4.38 \\
Work [21] & 7.22 \\
\hline
\end{tabular}




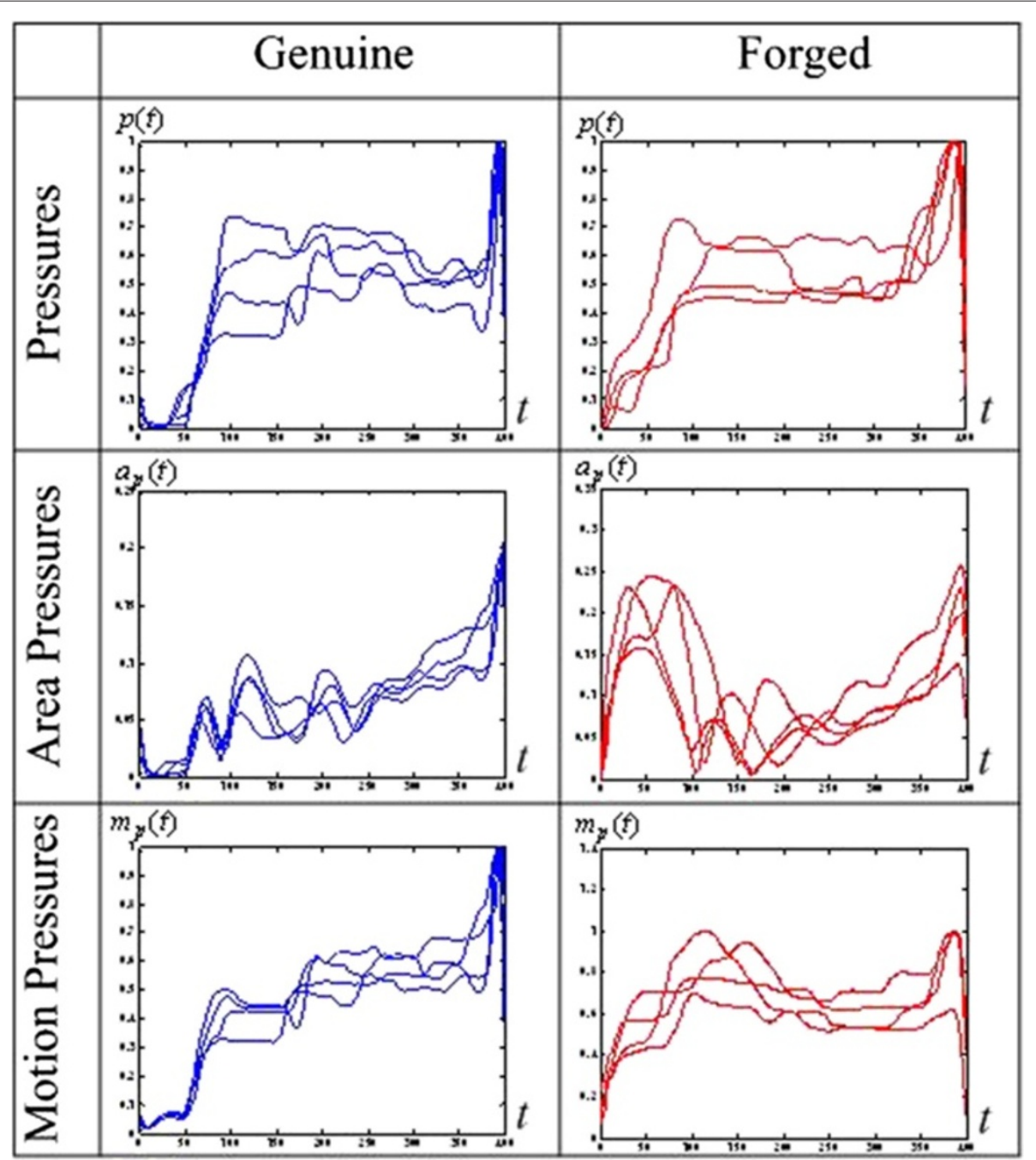

Figure 12 Original pen pressure, area pressure, and motion pressure obtained from the genuine signatures and forgeries.

The effectiveness of the two new handwriting features, motion pressure and area pressure, is shown in Figure 12. Figure 12 shows the plot of the original pen pressure, area pressure, and motion pressure obtained from genuine signatures and forgeries. It can be seen that the original pressure is not so different for genuine and forgery, but the area pressure and motion pressure proposed here are different for genuine and forgery because it is considered that the area pressure can characterize the information about the change of the shape and the pressure during signing process. In addition, the motion pressure can describe the information of the change of the pen-tip motion and the pressure during signing process.

\section{Conclusions}

In this paper, we proposed an online signature verification method based on the FIR system characterizing the time-frequency characteristics of dynamic handwriting features. The stable time-frequency characteristics of the dynamic feature were determined by selecting the suitable level of wavelet coefficients. The FIR system was realized by considering the selected wavelet coefficients of the dynamic features as the input and output of the system, respectively. The obtained impulse response was used as the individual feature for signature verification. It was found from our experiments that the proposed method is useful for online signature verification. In this research, a graphical tablet was used as the data acquisition system. Thus, the pen-point position was used to describe the handwriting feature in signing process. However, the graphical tablet can acquire such features as pen pressure and pen inclination. As such, the incorporation of such features into signature verification remains our future work. 


\section{Competing interests}

The authors declare that they have no competing interests.

\section{Acknowledgements}

This research was in part supported by the Thailand Research Fund (TRF) and the Commission on Higher Education (CHE) for research no. MRG5080378. The authors would like to express profound appreciation to J. Ortega-Garcia et al. for sharing the MCYT-100 public database and to W. Surakampontorn and P. Sooraksa for their kind and useful suggestions.

\section{Author details}

${ }^{1}$ Faculty of Engineering, King Mongkut's Institute of Technology Ladkrabang, Ladkrabang, Bangkok 10520, Thailand. ${ }^{2}$ School of Engineering, Tokai

University, 1117 Kitakaname, Hiratsuka, Kanagawa 259-1292, Japan.

\section{Received: 31 May 2012 Accepted: 25 November 2013}

Published: 11 December 2013

\section{References}

1. R Plamondon, G Lorette, Automatic signature verification and writer identification-the state of the art. Pattern Recognit. 22(2), 107-131 (1989)

2. F Leclerc, R Plamondon, Automatic signature verification: the state of the art-1989-1993. Int. J. Pattern Recognit. Artif. Intell. 8(3), 643-659 (1994)

3. D Impedovo, G Pirlo, Automatic signature verification: the state of art. IEEE Trans. Syst. Man Cybernatics-Part C: Appl. Rev. 38(5), 609-635 (2008)

4. M Faundez-Zanuy, Signature recognition state-of-the art. IEEE A \& E system magazine. 20(7), 28-32 (2005)

5. R Plamondon, SN Srihari, On-line and off-line handwriting recognition: a comprehensive survey. IEEE Trans. Pattern Anal. Mach. Intell. 22(1), 63-84 (2000)

6. M Yoshimura, Y Kato, S Matsuda, I Yoshimura, On-line signature verification incorporating the direction of pen movement. IEICE Trans. E74(7), 2083-2092 (1991)

7. P Zhao, A Higashi, Y Sato, On-line signature verification by adaptively weighted DP matching. IEICE Trans. E79-D(5), 535-541 (1996)

8. AK Jain, FD Griess, SD Connell, On-line signature verification. Pattern Recognit. 35, 2963-2972 (2002)

9. H Feng, C Choong Wah, Online signature verification using a new extreme points warping technique. Pattern Recognit. Lett. 24, 2943-2951 (2003)

10. K Huang, $\mathrm{H}$ Yan, Stability and style-variation modeling for on-line signature verification. Pattern Recognit. 36, 2253-2270 (2003)

11. M Faundez-Zanuy, On-line signature recognition based on VQ-DTW. Patt. Recognit. 40, 981-992 (2007)

12. B Ly Van, S Garcia-Salicetti, B Dorizzi, On using the Viterbi path along with HMM likelihood information for online signature verification. IEEE Trans. Syst. Man Cybernetics-part B 37(5), 1237-1247 (2007)

13. J Fierrez, J Ortega-Garcia, D Ramos, J Gonzalez-Rrodriguez, HMM-based on-line signature verification: feature extraction and signature modeling. Patt. Recognit. Lett. 28, 2325-2334 (2007)

14. P Thumwarin, T Matsuura, On-line writer recognition for Thai numeral IEICE Trans. E86-A(10), 2535-2541 (2003)

15. P Thumwarin, On-line writer recognition for Thai based on impulse response of FIR system characterizing handwriting motion, Thesis, Tokai University, 2004

16. P Thumwarin, T Matsuura, On-line writer recognition for Thai based on velocity of bary center of pen-point movement, in IEEE International Conference on Image Processing (Singapore, 24-27 October 2004), pp. 889-892

17. M Fuentes, S Garcia-Salicetti, B Dorizzi, On-line signature verification: fusion of hidden of Markov model and neural network via a support vector machine, in Proceedings of Eighth International Workshop on Frontiers in Handwriting Recognition (Canada, 6-8 August 2002), pp. 253-258

18. C Gruber, T Gruber, S Krinnger, B Sick, Online signature verification with support vector machines based on LCSS kernel functions. IEEE Trans. Syst. Man Cybernetics-part B 40, 1-4 (2009)

19. P Thumwarin, J Pernwong, N Wakayaphattaramanus, T Matsuura, On-line signature verification base on FIR System characterizing velocity and direction change of barycenter trajectory, in IEEE International Conference on Progress in Informatics and Computing (Shanghai, 10-12 December 2010), pp. 31-35

20. P Thumwarin, J Pernwong, T Matsuura, On-line signature verification base on FIR system characterizing motion pressure, in ACM SIGGRAPH Conference on Virtual-Reality Continuum and its Applications to Industry (Hong Kong, 11-12 December 2011), pp. 565-568

21. B Yanikoglu, A Kholmatov, Online signature verification using fourier descriptors. EURASIP J. Adv. Signal Process. doi:10.1155/2009/260516 (2009)

22. DZ Lejtman, E George, On-line handwritten signature verification using wavelets and back-propagation neural networks, in IEEE 6th International Conference on Document Analysis and Recognition (ICDAR 2001) (Seattle, 10-13 September 2001), pp. 992-996

23. R Jayadeven, S Subbaraman, PM Patil, Verification of hand printed signature images using discrete dyadic wavelet transform, in IEEE Second International Conference on Industrial and Information Systems (ICIIS 2007) (Penadeniya, 9-11 August 2007), pp. 314-345

24. K Wang, Y Wang, Z Zhang, On-line signature verification using wavelet packet, in IEEE International Joint Conference on Biometrics (IJCB) (Washington, DC, 11-13 October 2011), pp. 1-6

25. P Thumwarin, S Khem, K Janchitraponvej, T Matsuura, On-line writer dependent character recognition for Khmer based on FIR system characterizing handwriting motion, in 5th International Conference on Electrical Engineering/Electronics, Computer, Telecommunications and Information Technology (ECTI-CON 2008), vol. 1 (Krabi, 14-17 May 2008), pp. 541-544

26. J Ortega-Garcia, J Fierrez-Augilar, D Simon, J Gonzalez, M Faundez-Zanuy, V Espinosa, A Satue, I Hernaez, J-J Igarza, C Vivaracho, D Escudero, Q-I Moro, MCYT baseline corpus: a bimodal biometric database. IEEE Proc. Vis. Image Signal Process 150(6), 395-401 (2003)

\section{doi:10.1186/1687-6180-2013-183}

Cite this article as: Thumwarin et al.: FIR signature verification system characterizing dynamics of handwriting features. EURASIP Journal on Advances in Signal Processing 2013 2013:183.

\section{Submit your manuscript to a SpringerOpen ${ }^{\circ}$ journal and benefit from:}

- Convenient online submission

Rigorous peer review

- Immediate publication on acceptance

- Open access: articles freely available online

- High visibility within the field

- Retaining the copyright to your article

Submit your next manuscript at $>$ springeropen.com 